\title{
Acute Cholangitis Secondary to a Clogged Biliary Stent: A Review on the Cause of Clogging and the Appropriate Time of Replacement
}

\author{
Ahmad Ramzi Yusoffa Qamarina Zettie Dyana Kamarul Anuar ${ }^{a}$ \\ Shahril Khalid $^{\mathrm{a}}$ Suryati Mokhtar ${ }^{\mathrm{b}}$ \\ aDepartment of Surgery, Faculty of Medicine, Universiti Teknologi MARA (UiTM), Selangor, \\ Malaysia; 'Department of Hepatobiliary Surgery, Selayang Hospital, Selangor, Malaysia
}

Keywords

Choledocholithiasis · Acute cholangitis · Gallstone

\begin{abstract}
Choledocholithiasis is preferably treated by endoscopic retrograde cholangiopancreatography (ERCP) with sphincterotomy and stone removal, to reduce the risk for acute cholangitis. Frequently, patients who are ill, surgically unfit, or unable to undergo stone extraction during the index procedure underwent antibacterial treatment and temporary biliary stenting via ERCP to prevent biliary sepsis and septic shock. After a period of convalescence, a repeat ERCP is scheduled to clear the bile duct and remove the stent, followed by laparoscopic cholecystectomy to complete the treatment circuit. Cholangitis may often recur in patients with an indwelling biliary stent while waiting for definitive treatment. Here, we present a case of a 42-year-old female with choledocholithiasis who developed moderate acute cholangitis 5 months after ERCP and insertion of a biliary plastic stent. She was provisionally diagnosed with obstructive jaundice with concurrent acute cholecystitis. Through intravenous antibacterial therapy, stent exchange, and an interval open cholecystectomy, she had fully recovered. We also discuss the underlying mechanism of stent blockage and the optimal interval for stent exchange after temporary placement for benign cases. Understanding the pathophysiology of stent clogging and recognizing the optimal interval for stent replacement may help reduce the risk of stent clogging and potentially fatal acute cholangitis.
\end{abstract}




\section{Introduction}

Acute cholangitis is a severe bile duct inflammation frequently caused by choledocholithiasis, which is due to gallstone migration into the bile duct [1]. Benign or malignant biliary strictures, parasitic infestation, and previous bilioenteric anastomosis can cause acute cholangitis [1]. Gallstones have been implicated in $28-70 \%$ of acute cholangitis cases, and clinical manifestations such as abdominal pain, jaundice, fever, and tender hepatomegaly indicate a surgical emergency $[1,2]$. It requires immediate treatment with intravenous antibacterial therapy and stone extraction via endoscopic retrograde cholangiopancreatography (ERCP); if it fails, a temporary biliary stent is placed to relieve the obstruction [2,3]. Ironically, patients with a biliary stent are prone to recurrent cholangitis while waiting for a definitive treatment. Herein, we present a case of a 42-year-old female who developed moderate acute cholangitis 5 months after insertion of an indwelling biliary stent. Blood investigation and imaging revealed obstructive jaundice with concurrent acute cholecystitis. Nonetheless, antibacterial therapy, stent exchange, and an interval open cholecystectomy a few months later led to successful treatment. Furthermore, we conducted a literature review to understand the underlying mechanism of stent blockage and the optimal interval for stent exchange after index insertion to avoid the risks of clogging and cholangitis.

\section{Case Report}

A 42-year-old female presented to our hospital with 3-day progressive and recurrent right upper quadrant pain. The pain radiated to the back and accompanied by pyrexia, jaundice, nausea, and vomiting. She had dark urine and pale stools but no anorexia or weight loss. She does not smoke or consume alcohol. She experienced similar manifestations 5 months prior and underwent ERCP at a referring hospital by the gastroenterologist. Choledocholithiasis was confirmed. After endoscopic sphincterotomy and balloon sweep, several common bile duct (CBD) stones were partially extracted; thus, insertion of a biliary plastic stent was required to prevent recurrent cholangitis. She was then scheduled for another ERCP and elective cholecystectomy a few months later at our center; however, these procedures were delayed because of the rescheduling of operating theatre listing.

Physical examination revealed that the patient appeared unwell, dehydrated, and jaundiced, with a body mass index of $20.3 \mathrm{~kg} / \mathrm{m}^{2}$. Although her blood pressure, respiratory rate, and oxygen saturation were normal $(111 / 55 \mathrm{~mm} \mathrm{Hg}, 19$ breaths per minute, and $98 \%$ on 3 $\mathrm{L} / \mathrm{min}$ nasal cannula oxygen), she was febrile and tachycardic $\left(38.4^{\circ} \mathrm{C}\right.$ and 110 beats per minute). Furthermore, she exhibited abdominal tenderness, guarding in the right upper quadrant, and a positive Murphy's sign, with no ascites but with audible bowel sounds.

Laboratory findings included elevated levels of white blood cells $\left(12.05 \times 10^{9} / \mathrm{L}\right)$ and serum bilirubin $(119 \mu \mathrm{mol} / \mathrm{L})$; meanwhile, the alkaline phosphatase and alanine amino transaminase levels were 38 and $30 \mathrm{U} / \mathrm{L}$, respectively. Abdominal radiograph demonstrated an indwelling biliary stent with several radio-opaque calculi shadows in the right upper quadrant (Fig. 1). In contrast, abdominal ultrasound and computed tomography (CT) scans revealed a grossly distended gallbladder containing sludge and several gallstones (Fig. 2, 3). The largest stone measured $1.6 \mathrm{~cm}$ in diameter. Additionally, a $1.2 \times 1.4 \mathrm{~cm}$ stone was found in the cystic duct. The CT scan further showed diffuse thickening of the gallbladder wall (approximately $1.0 \mathrm{~cm}$ thick), with pericholecystic fluid and streakiness of adjacent mesenteric fat (Fig. 2). We found no perforation in the absence of an air locule adjacent to the distended gallbladder or a gallbladder wall defect. Therefore, the patient obtained a provisional diagnosis of obstructive jaundice secondary to moderate acute cholangitis with concurrent acute cholecystitis.

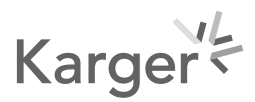


Fig. 1. Abdominal radiograph showing an indwelling biliary stent with several radio-opaque calculi shadows in the right upper quadrant.
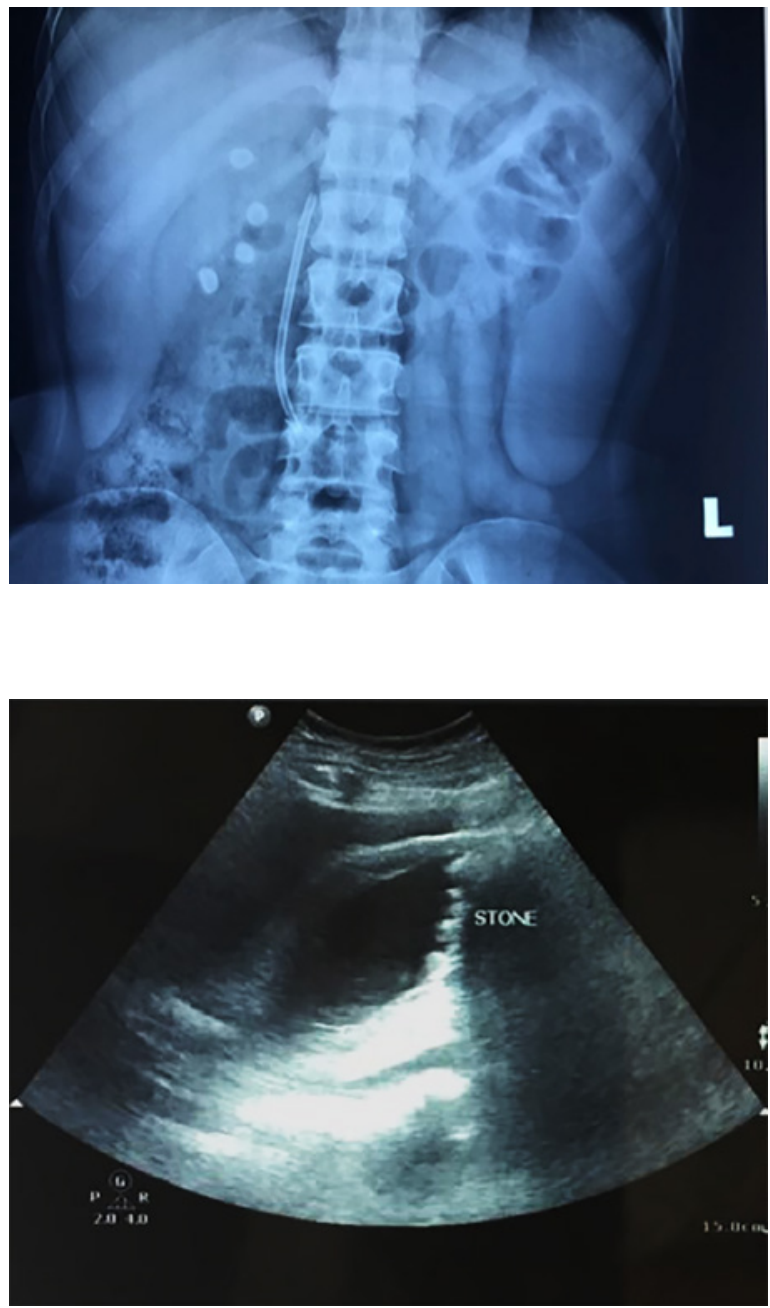

gallstones.

Fig. 3. Axial CT abdominal scan showing a grossly distended gallbladder with multiple calculi and sludge within. Pericholecystic fluids are present in the gallbladder surrounding.

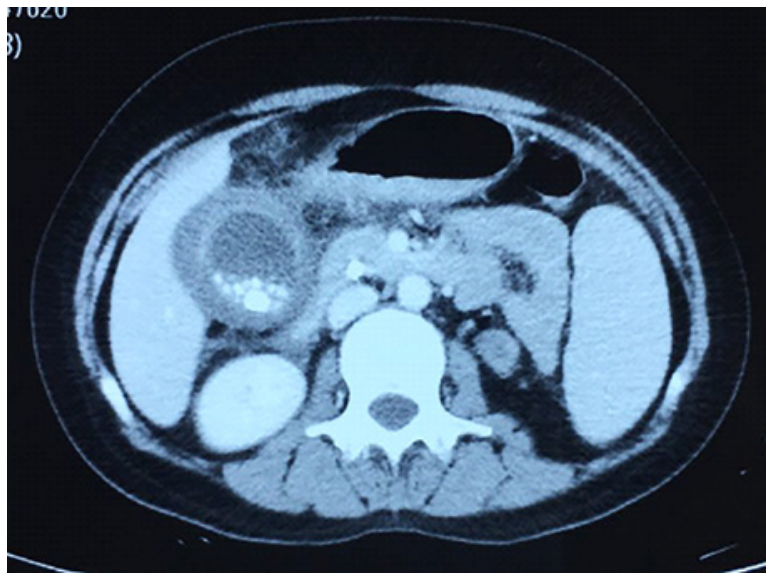

Subsequently, she began receiving $4.5 \mathrm{~g}$ of piperacillin/tazobactam and $50 \mathrm{mg}$ of hydrocortisone both intravenously for four times a day in the ward and was kept nil by mouth. The following day, ERCP revealed a clogged biliary stent, resulting in the accumulation of pus 
streaking down the ampulla. Considering the occurrence of moderate cholangitis, we decided to shorten the procedure time by not completely removing all the CBD stones; instead, we replaced the stent with another plastic stent measuring $10 \mathrm{Fr}$ in diameter and $10 \mathrm{~cm}$ in length. Thereafter, she was transferred to the high-dependency ward for close observation and antibacterial therapy. Consequently, she had fully recovered and was discharged 3 days later on an oral amoxicillin/clavulanate regimen. After 1 month, the remnant stones and stent were removed altogether by repeat ERCP. After 6 months, she underwent elective laparoscopic cholecystectomy, which had to be converted into open surgery because of the obliteration of Calot's triangle during laparoscopy.

\section{Discussion}

Cholelithiasis is prevalent in Malaysia, with a prevalence rate of $16.83 \%$ in females and only $5.36 \%$ in males [4]. Choledocholithiasis may complicate gallstone disease, causing obstructive jaundice, cholangitis, acute pancreatitis, and eventually, biliary stricture and secondary biliary cirrhosis. Given the possibility of serious complications in choledocholithiasis, definitive treatment with ERCP, sphincterotomy, and stone clearance followed by cholecystectomy is generally required $[1,2]$. When biliary stones are not completely cleared during ERCP, a biliary plastic stent should be temporarily inserted to act as a conduit for bile outflow and avoid bile obstruction and stone impaction, which will lead to cholangitis if left unmanaged $[2,5]$. Our patient underwent biliary stent placement during the index ERCP at the referring hospital to prevent such complications.

However, as its primary disadvantage, biliary stent placement can possibly cause blockage a few days or several months after insertion. Thus, scheduled stent exchanges are needed to avoid blockage and recurrent cholangitis if the definitive procedure cannot be arranged on time. Our patient's repeat ERCP at our center for complete stone clearance and later cholecystectomy were delayed for 5 months because her operation date was rescheduled to accommodate more urgent cancer cases. Unfortunately, she presented with classic Charcot's triad while waiting for surgery, presumably resulting from acute cholangitis. Her differential diagnoses at presentation included acute cholecystitis, gallbladder empyema, acute pancreatitis, pyogenic liver abscess, and hepatitis. To confirm the diagnosis, we performed routine blood investigations, an initial transabdominal ultrasound, and a contrast-enhanced CT scan of the abdomen.

Based on the investigation results, a provisional diagnosis of moderate acute cholangitis (Grade II) secondary to the blocked biliary stent with concurrent acute cholecystitis was made, given that no organ failures were involved $[2,6]$. She then started receiving $\beta$-lactam/ $\beta$-lactamase inhibitor combinations for antibacterial treatment. Then, she underwent ERCP and stent change as a form of internal drainage to alleviate her cholangitis. This treatment strategy was based on her history of stenting per the TG13 guideline, which mandates early endoscopic or percutaneous drainage for acute cholangitis [2]. Emergency cholecystectomy and CBD exploration by laparoscopy would be technically demanding, and an open surgery theoretically causes complications during an acute infectious setting. Definitive surgery is best performed after the patient's general condition has improved [2]. More commonly, after replacing the blocked stent and administering the correct antibacterial therapy, the acute condition improves, as seen in our patient. A few months later, once the inflammatory process had resolved, laparoscopic cholecystectomy was performed. It was hindered, however, by the difficulty of gaining a critical view of safety, which necessitated open surgery.

Stent blockage believed to be caused by duodenobiliary reflux, that is, the regression of duodenal contents into the biliary system, resulting from endoscopic sphincterotomy, which breaks down the sphincter of Oddi [7]. This causal role is supported by microscopic findings

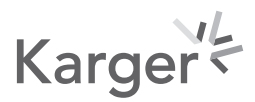


Table 1. Summary of clinical studies determining the time to stent occlusion and the rate of stent patency, published over the last 2 decades

\begin{tabular}{llllll}
\hline Author & Year & Study design & Patients, $n$ & $\begin{array}{l}\text { Median stent survival } \\
\text { (time to occlusion), months }\end{array}$ & Patency rate, \% \\
\hline Weickert et al. [7] & 2001 & Retrospective & 100 & 3 & NA \\
Donelli et al. [9] & 2006 & Review & - & $4-5$ & NA \\
Li et al. [15] & 2009 & Retrospective & 50 & 12 & 94 (6 months), \\
& & & & & 79 (12 months), \\
& & & 78 & 3 & NA (24 months) \\
Di Giorgio et al. [14] & 2013 & RCT & 3 & 100 (3 months) \\
Ye et al. [3] & 2016 & Retrospective & 64 & $3-6$ & NA \\
Vaishnavi et al. [11] & 2018 & Prospective & 81 & 12 & 91.4 (6 months), \\
Tohda and Dochin [5] & 2018 & Retrospective & 87 & & 88.6 (12 months) \\
& & & & &
\end{tabular}

RCT, randomized controlled trial; NA, not available.

of dietary fiber, bile, bacteria, fibrin, debris, granulation tissue, and inflammatory cells detected in the clogging material from retrieved stents [7]. Additionally, the body considers a stent as a foreign body in the biliary system, triggering bacterial growth and bile infection via ascending infection [8]. Consequently, inflammation (cholangitis) and even brown pigment stone (calcium bilirubinate) formation will eventually occur [5], as evidenced by the increased viscosity of infected bile. With increased viscosity, bile flow velocity is reduced, leading to bile stasis and increased bile salt precipitation by the bacteria [9]. Furthermore, bacterial colonization may result in biofilm formation within the biliary stent, further causing occlusion $[9,10]$. This biofilm formation is a three-step process that begins with priming the stent surface with various microbial proteins, then microbial adhesion to the stent, and finally, forming an exopolysaccharide matrix to embed the microbial colonies and other particles into mature biofilm $[9,10]$. Biofilm formation is a time-dependent process, and the risk of biliary plastic stent occlusion increases gradually after 3 months $[9,11]$.

Moreover, the sludge formed by the occluded stent showed a significantly higher rate of infection by Gram-negative bacteria than by Gram-positive bacteria [11]. Hence, a broadspectrum antibacterial was required at the initial presentation of this patient. Escherichia coli is present in $71 \%$ of concurrent bile and blood cultures, followed by Klebsiella spp. in 53\%, Enterococcus spp. in 35\%, and Streptococcus spp. in 27\% [12], almost similar to other reports $[9,11]$. In the report of Lübbert et al. [13], the most prevalent bacteria were Enterococcus spp. (79.3\%) and Candida spp. (55.9\%), which are significantly associated with prolongation in indwelling stent time.

Unfortunately, recommendation for stent exchange interval after a failed initial stone clearance still has not achieved consensus, considering that prolonged indwelling stent time might lead to late complications, such as acute cholangitis from stent occlusion, biliary obstruction, and biliary-related mortality. However, Di Giorgio et al. [14], in their randomized controlled trial of 78 patients, concluded that stent changes after 3 months or sooner significantly reduced the risk of acute cholangitis compared with stent changes on demand. Similarly, Ye et al. [3] reported that insertion of two plastic stents demonstrated a higher patency rate at 3 months than only one stent. These findings are in line with the respective studies of Donelli et al. [9] and Vaishnavi et al. [11]; they reported that after 3 months, the risk of clogging standard biliary stents increases progressively, as well as the risk of biofilm formation and occlusion (Table 1).

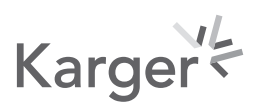


Nonetheless, several studies have demonstrated that plastic stents generally remain patent at 6 months, eliminating the need for stent change at 3 months and extending the indwelling time to 12 months. In the study by Li et al. [15], the stent patency began to deteriorate rapidly after 6 , 12 , and 24 months at a rate of 94\%, 79\%, and 58\%, respectively. Similarly, Tohda and Dochin [5] discovered that $91.4 \%$ of biliary plastic stents are still patent at 6 months compared with $88.6 \%$ at 12 months. Although these studies concluded that changing the stent at 3 months is unnecessary, keeping the stent longer or changing it on demand is perhaps only suitable for older patients with a high risk, patients with severe comorbidities, and patients who are surgically unfit or who simply decline further procedure albeit with the inherent risk of cholangitis $[3,5,15]$.

In conclusion, ERCP and temporary biliary stenting are the optimal treatment for acute cholangitis secondary to choledocholithiasis to prevent sepsis. However, the risk of developing recurrent cholangitis should be explained on the patient despite having an indwelling stent. Prolonged placement of biliary plastic stents increases the risk of clogging caused by biofilm formation, bacterial colonization, and sludge deposition. Scheduled stent change every 3 months reduces the risk of developing cholangitis for a fit patient with benign disease awaiting definitive treatment, which could be either second ERCP and stone extraction or surgery.

\section{Acknowledgments}

The authors would like to thank the Director General of Health Malaysia and the Dean of the Faculty of Medicine, Universiti Teknologi MARA, Malaysia, for their permission to publish this manuscript.

\section{Statement of Ethics}

Written informed consent was obtained from the patient for publication of the details of their medical case and any accompanying images. This study protocol was reviewed, and the need for approval was waived by the Research Ethics Committee Meeting \#9/2021. The meeting was chaired by Associate Professor Dr. Thuhairah Hasrah Abdul Rahman from the Faculty of Medicine, Universiti Teknologi MARA, Selangor, Malaysia.

\section{Conflict of Interest Statement}

The authors have no conflicts of interest to declare.

\section{Funding Sources}

This research did not receive any grant or funding from any agency.

\section{Author Contributions}

Ahmad Ramzi Yusoff co-written the draft, provided the literature review, and supervised the research project. Qamarina Zetti Dyana Kamarul Anuar collected the patient's clinical data and wrote the first draft. Shahril Khalid revised and critically appraised the manuscript. Suryati Mokhtar managed and treated the patient and approved the final manuscript. 


\section{Data Availability Statement}

All data generated or analyzed during this study are included in this article. The accompanying images are retrievable from the hospital information system with written permission. Further enquiries can be directed to the corresponding author.

\section{References}

1 Kimura Y, Takada T, Kawarada Y, Nimura Y, Hirata K, Sekimoto M, et al. Definitions, pathophysiology, and epidemiology of acute cholangitis and cholecystitis: Tokyo guidelines. J Hepatobiliary Pancreat Surg. 2007 Jan; 14(1):15-26.

2 Miura F, Takada T, Strasberg SM, Solomkin JS, Pitt HA, Gouma DJ, et al. TG13 flowchart for the management of acute cholangitis and cholecystitis. J Hepatobiliary Pancreat Sci. 2013 Jan;20(1):47-54.

3 Ye X, Huai J, Sun X. Effectiveness and safety of biliary stenting in the management of difficult common bile duct stones in elderly patients. Turk J Gastroenterol. 2016 Jan;27(1):30-6.

4 Sood S, Winn T, Ibrahim S, Gobindram A, Arumugam AA, Razali NC, et al. Natural history of asymptomatic gallstones: differential behaviour in male and female subjects. Med J Malaysia. 2015 Dec;70(6):341.

5 Tohda G, Dochin M. Management of endoscopic biliary stenting for choledocholithiasis: evaluation of stentexchange intervals. World J Gastrointest Endosc. 2018 Jan;10(1):45.

6 Yokoe M, Takada T, Strasberg SM, Solomkin JS, Mayumi T, Gomi H, et al. New diagnostic criteria and severity assessment of acute cholecystitis in revised Tokyo guidelines. J Hepatobiliary Pancreat Sci. 2012 Sep; 19(5): 578-85.

7 Weickert U, Venzke T, König J, Janssen J, Remberger K, Greiner L. Why do bilioduodenal plastic stents become occluded? A clinical and pathological investigation on 100 consecutive patients. Endoscopy. 2001 Sep;33(9): 786-90.

8 Odabasi M, Arslan C, Akbulut S, Abuoglu HH, Ozkan E, Yildiz MK, et al. Long-term effects of forgotten biliary stents: a case series and literature review. Int J Clin Exp. 2014 Aug 15;7(8):2045-52.

9 Donelli G, Guaglianone E, Di Rosa R, Fiocca F, Basoli A. Plastic biliary stent occlusion: factors involved and possible preventive approaches. Clin Med Res. 2007 Mar;5(1):53-60.

10 Guaglianone E, Cardines R, Vuotto C, Di Rosa R, Babini V, Mastrantonio P, et al. Microbial biofilms associated with biliary stent clogging. FEMS Immunol Med Microbiol. 2010 Aug;59(3):410-20.

11 Vaishnavi C, Samanta J, Kochhar R. Characterization of biofilms in biliary stents and potential factors involved in occlusion. World J Gastroenterol. 2018 Jan;24(1):112.

12 Schneider J, Hapfelmeier A, Fremd J, Schenk P, Obermeier A, Burgkart R, et al. Biliary endoprosthesis: a prospective analysis of bacterial colonization and risk factors for sludge formation. PLoS One. 2014 Oct; $9(10)$ : e110112.

13 Lübbert C, Wendt K, Feisthammel J, Moter A, Lippmann N, Busch T, et al. Epidemiology and resistance patterns of bacterial and fungal colonization of biliary plastic stents: a prospective cohort study. PLoS One. 2016 May; 11:e0155479.

14 Di Giorgio P, Manes G, Grimaldi E, Schettino M, D’Alessandro A, Di Giorgio A, et al. Endoscopic plastic stenting for bile duct stones: stent changing on-demand or every 3 months. A prospective comparison study. Endoscopy. 2013 Dec;45(12):1014-7.

15 Li KW, Zhang XW, Ding J, Chen T, Wang J, Shi WJ. A prospective study of the efficacy of endoscopic biliary stenting on common bile duct stones. J Dig Dis. 2009 Nov;10(4):328-31. 